\title{
Lower Cranial Nerve Palsies in the COVID-19 Pandemic: A 10-Case Series of Intensive Care Unit Patients
}

\author{
Pierre Decavel ${ }^{a, b}$ Olympe Nahmias ${ }^{c}$ Carine Petit ${ }^{c}$ Laurent Tatu ${ }^{b, d}$ \\ aDepartment of Rehabilitation, Hôpital Fribourgeois, Villars-sur-Glâne, Switzerland; b ${ }^{\text {EA }} 481$ Integrative and Clinical \\ Neuroscience, University of Franche-Comté, Besançon, France; 'Department of Rehabilitation, CHRU de Besançon, \\ Besançon, France; d Department of Neuromuscular Diseases and Department of Anatomy, CHRU Besançon, \\ University of Franche-Comté, Besançon, France
}

\section{Keywords \\ Lower cranial nerve palsy · COVID-19 · Intensive care unit}

\begin{abstract}
Introduction: A number of neurological complications of COVID-19 have been identified, including cranial nerve paralyses. We present a series of 10 patients with lower cranial nerve involvement after severe COVID-19 infection requiring hospitalization in an intensive care unit. Methods: We conducted a retrospective, observational study of patients admitted to the post-intensive care unit ( $p$-ICU) of Besançon University Hospital (France) between March 16 and May 22, 2020. We included patients with confirmed COVID-19 and cranial neuropathy at admission to the p-ICU. All these patients were treated by orotracheal intubation, and all but one underwent prone-position ventilation therapy. Results: Of the 88 patients admitted to the p-ICU, 10 patients (11\%) presented at least 1 cranial nerve palsy. Of these 10 patients, 9 had a hypoglossal nerve palsy and 8 of these also had a deficit in another cranial nerve. The most frequent association was between hypoglossal and vagal palsies (5 patients). None of the patients developed neurological signs related to a global neuropathy. We found no correlation between the intensity of the motor limb weakness and the occurrence of
\end{abstract}

lower cranial nerve palsies. All but 2 of the patients recovered within less than a month. Conclusion: The mechanical compressive hypothesis, linked to the prone-position ventilation therapy, appears to be the major factor. The direct toxicity of SARS-CoV-2 and the context of immune dysfunction induced by the virus may be involved in a multifactorial etiology.

(c) 2021 S. Karger AG, Base

\section{Introduction}

The COVID-19 virus that began to circulate in late 2019 has been shown to induce some neurological complications, such as cranial nerve paralyses including oculomotor nerve dysfunction [1-3]. The precise mechanisms of these nerve injuries are still debated and could be attributed to direct viral infiltration of the nervous system, autoimmune response, or a compressive disorder [4, 5]. In COVID-19 patients admitted to an intensive care unit (ICU), prolonged orotracheal intubation associated with use of prone-position ventilation therapy is the primary cause of lower cranial neuropathies [2].

In this article, we present a series of 10 patients with lower cranial nerve involvement after a prolonged stay in karger@karger.com www.karger.com/ene

Karger
(C) 2021 S. Karger AG, Basel
Correspondence to:

Laurent Tatu, laurent.tatu@ univ-fcomte.fr 
an ICU due to COVID-19 infection. We discuss the potential mechanisms underlying these nerve injuries. It is crucial to consider how to prevent patients from developing this kind of complication. Moreover, early detection of these cranial nerve paralyses could reduce the duration of hospitalization.

\section{Materials and Methods}

A retrospective observational study was conducted in the postintensive care unit (p-ICU) of Besançon University Hospital (France) between March 16 and May 22, 2020, during the COVID-19 pandemic. The patients were admitted to the p-ICU after a stay in the ICU.

Of the p-ICU patients with confirmed COVID-19, those presenting with cranial neuropathy at admission were included in this study. Their acute respiratory distress syndrome had been treated in the ICU by orotracheal intubation and prone-position ventilation therapy. They were examined once per day with a systematic procedure including a complete cranial nerve examination and an evaluation of the strength of 3 muscle groups in each upper and lower limb, measured by the Medical Research Council sum score (MRC-SS) [6]. Swallowing disorders were also regularly evaluated. In the event of persistent dysphagia, a nasofibroscopy was performed.

\section{Results}

Of the 88 patients admitted to the p-ICU during the study period, the 10 patients (11\%) who presented at least 1 cranial nerve palsy were included in this study. The general characteristics of the 10 patients are given in Table 1 . At admission to the p-ICU, 9 had a hypoglossal nerve palsy. Of these 9 patients, 8 had a deficit in at least 1 other cranial nerve: vagal nerve (7/8), facial nerve (2/8), and glossopharyngeal nerve $(2 / 8)$. The most frequent combination was a hypoglossal and a vagal palsy (5 patients). The last patient had an isolated vagus nerve palsy. No oculomotor nerve dysfunction was observed.

The patients were all male. They were admitted to the p-ICU between $24 \mathrm{~h}$ and 5 days after being extubated. One patient (patient 3 ) was admitted 6 days after completion of a tracheostomy. Prone-position ventilation therapy was performed in 9 of the 10 patients: $1-11$ sessions of 16 consecutive hours.

At admission to the p-ICU, all the patients had at least 1 criterion of undernutrition (weight loss or low serum albumin). Three patients had a severe muscular limb weakness and 5 had a significant muscular limb weakness according to the MRC-SS (Table 1). Three patients had a facial pressure sore. In 1 patient with a facial palsy, the

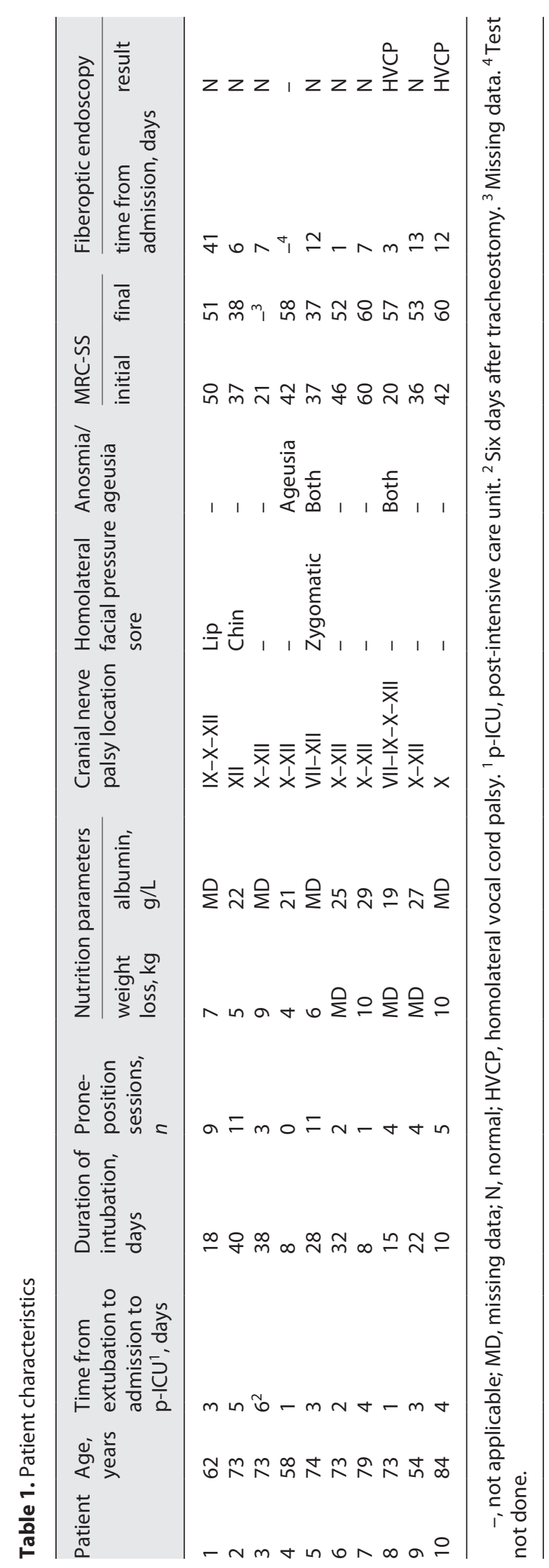

Eur Neurol 2022;85:136-139 
facial pressure sore was located on the course of the facial nerve.

None of the patients developed neurological signs related to an acute neuropathy. The limb motor weakness was linked to myopathy acquired in the ICU. There was no clear correlation between the intensity of the motor limb weakness and the occurrence of lower cranial nerve palsies.

The patients were re-evaluated 1 month after leaving the p-ICU. At that time, $8 / 10$ had a normal cranial nerve examination and 2/10 still had a hypoglossal nerve palsy.

\section{Discussion}

In the spring of 2020, during the COVID-19 pandemic, we observed an unexpectedly high rate of lower cranial nerve palsies in patients admitted to our p-ICU. The case study of one of these patients (patient 1), who developed Tapia's syndrome, has already been published [2]. Following that first case, we carefully and systematically studied cranial nerve dysfunction in patients admitted to our p-ICU, allowing us to present the results of this 10-patient series. In this series, some clinical associations of cranial nerve dysfunction could be regarded as close to classic cranial nerve syndromes such as Tapia's syndrome. Nevertheless, we preferred to use a purely clinical descriptive approach. The hypoglossal nerve was the most frequently involved, and the most frequent association was hypoglossal and vagus nerve dysfunction.

From our point of view, mechanical compression, linked to the prone-position ventilation therapy, appears to be the major factor. During their stays in the ICU, all but one of the patients had been regularly placed in a completely prone position. This kind of positioning has already been described as an etiological factor of nerve compression, in particular for the hypoglossal nerve [7]. In our series, this compressive mechanism hypothesis is supported by the patients' rapid recovery, which suggests a neurapraxia frequently observed in compressive nerve disorders. Four patients developed facial pressure sores, which also supports the hypothesis of a compressive mechanism. All the patients included in this study were male. The morphology of the male hyoid bone seems to be a risk factor for local compressive lower cranial nerve palsies such as those we observed [8].

Another etiological factor could be the neurotropism of SARS-CoV-2 [4]. Cranial nerve involvement may reflect the neurovirulence of the virus. Some facial or oculomotor nerve palsies have been reported and linked to viral toxicity [1]. However, in our series, no oculomotor dysfunction was observed, and at least 1 facial paralysis may be explained by a facial sore.

The context of immune dysfunction induced by SARSCoV-2 without direct neurotoxicity of the virus could also be discussed. In many countries, an unexplained number of instances of Guillain-Barré syndrome were recorded in patients with negative SARS-CoV-2 nasopharyngeal swabs and negative IgM and IgG SARS-CoV-2 serology $[9,10]$.

A context of immune dysfunction could be a precipitating factor for nerve paralysis. A multifactorial etiology cannot be definitively ruled out in our patients. Nevertheless, the mechanical factor seems to be clearly predominant as the compressive factor triggers the nerves, which are weakened by other conditions. This study, which focuses on unexpected complications, has some limitations inherent to a retrospective observational study. Because all patients were treated with anticoagulant therapy, it was not feasible to perform electromyography, which would have enabled us to evaluate the severity of the cranial nerve palsies and further discuss their etiological mechanism. Due to the anticoagulant treatment, the cerebrospinal fluid was not routinely analyzed. Fiberoptic endoscopic evaluation was difficult to achieve and was not carried out systematically. Some of these examinations were performed too late, which explains numerous normal results.

The findings exposed in this article should lead us to reflect on patient management, with the aim of limiting complications as much as possible. One effective procedure is the systematic use of an adapted cushion to control the position of the head during prone-position ventilation therapy [7]. Corticosteroid treatment may be of interest as it has been shown to be effective in the context of compressive Tapia's syndromes [11]. In addition, systematic screening for swallowing disorders using adapted protocols will limit the consequences of lower cranial nerve paralysis [12].

\section{Acknowledgment}

The authors thank Jennifer Dobson for proofreading the manuscript.

\section{Statement of Ethics}

According to the French Regulatory Authority for clinical studies, prospective and retrospective studies with observational analysis only are not evaluated by Human Protection Committees. The study had this study design and received no opposing statement 
from the local ethics committee (Human Protection Committee East Area II, Besançon). According to the same regulatory authority, written informed consent is not required for this type of retrospective and observational study. The patients or their guardians have been informed of the use of their data for the study. We received no statements of opposition.

\section{Conflict of Interest Statement}

The authors have no conflicts of interest to declare.

\section{Funding Sources}

This study received no funding.

\section{Author Contributions}

Pierre Decavel contributed to acquisition of data and writing. Olympe Nahmias contributed to acquisition of data and drafting. Carine Petit contributed to acquisition of data and drafting. Laurent Tatu contributed to writing and critical review of the manuscript.

\section{Data Availability Statement}

All data generated or analyzed during this study are included in this article. Further enquiries can be directed to the corresponding author.

\section{References}

1 Dinkin M, Gao V, Kahan J, Bobker S, Simonetto M, Wechsler P, et al. Author response: COVID-19 presenting with ophthalmoparesis from cranial nerve palsy. Neurology. 2020; 95(5):411-3.

2 Decavel P, Petit C, Tatu L. Tapia syndrome at the time of the COVID-19 pandemic: lower cranial neuropathy following prolonged intubation. Neurology. 2020;95(7):312-3.

3 Gutiérrez-Ortiz C, Méndez-Guerrero A, Rodrigo-Rey S, San Pedro-Murillo E, BermejoGuerrero L, Gordo-Mañas R, et al. Miller Fisher syndrome and polyneuritis cranialis in COVID-19. Neurology. 2020;95(5):e601-5.

4 Li YC, Bai WZ, Hashikawa T. The neuroinvasive potential of SARS-CoV2 may play a role in the respiratory failure of COVID-19 patients. J Med Virol. 2020;92(6):552-5.
5 Costello F, Dalakas MC. Cranial neuropathies and COVID-19: neurotropism and autoimmunity. Neurology. 2020;95(5):195-6.

6 Stevens RD, Marshall SA, Cornblath DR, Hoke A, Needham DM, de Jonghe B, et al. A framework for diagnosing and classifying intensive care unit-acquired weakness. Crit Care Med. 2009;37(10 Suppl):S299-308.

7 Shah AC, Barnes C, Spiekerman CF, Bollag LA. Hypoglossal nerve palsy after airway management for general anesthesia: an analysis of 69 patients. Anesth Analg. 2015;120(1):105-20.

8 Urbanová P, Hejna P, Zátopková L, Šafr M. The morphology of human hyoid bone in relation to sex, age and body proportions. Homo. 2013;64(3):190-204.
9 Tatu L, Nono S, Grácio S, Koçer S. GuillainBarré syndrome in the COVID-19 era: another occasional cluster? J Neurol. 2021;268(4): 1198-200.

10 Gigli GL, Bax F, Marini A, Pellitteri G, Scalise A, Surcinelli A, et al. Guillain-Barré syndrome in the COVID-19 era: just an occasional cluster? J Neurol. 2021;268(4):1195-7.

11 Boğa I, Aktas S. Treatment, classification, and review of Tapia syndrome. J Craniofac Surg. 2010;21(1):278-80.

12 De Luca P, Cavaliere M, Scarpa A, Savignano L, Cassandro E, Cassandro C, et al. Rehabilitation protocol for unilateral laryngeal and lingual paralysis (Tapia syndrome). Ear Nose Throat J. 2021;100:734S-7S. 